\title{
Potassium Aggravated Myotonia
}

National Cancer Institute

\section{Source}

National Cancer Institute. Potassium Aggravated Myotonia. NCI Thesaurus. Code C122788.

A group of autosomal dominant inherited non-dystrophic myotonias caused by mutations of the SCN4A gene, resulting in sodium muscle channelopathy. They are characterized by muscle stiffness, which worsens by ingestion of potassium-rich food. This group includes myotonia fluctuans, myotonia permanens, and acetazolamideresponsive myotonia. 